\title{
Desarrollo de la competencia transversal de "Análisis y Resolución de Problemas” en la asignatura Centrales Nucleares Avanzadas
}

\section{Sergio Gallardo y Sofía Carlos}

Departamento de Ingeniería Química y Nuclear, Universitat Politècnica de València.

\begin{abstract}
The course "Advanced Nuclear Power Plants" is taught in the $4^{\text {th }}$ year of the Grade of Energy Engineering, in the Universitat Politècnica de València. This is the first year this course have been taught and has a reduced number of students enrolled, as it is offered as an optional subject. All the students have a prior training in Nuclear Energy through the subject "Nuclear Technology". In this course, design and safety analysis of new nuclear fission reactors is studied. During this course it has begun work on the transversal skill "Problem Analysis and Resolution" in both theory and practice sessions. Aware of the importance of the development of this skill, different sessions are designed to solve a complex problem (not exercises) in which students should be involved in decision-making processes. For this purpose, the calculation of different design parameters of an advanced nuclear reactor using a Monte Carlo code is proposed. The problem to solve is open and not completely defined, and the class sessions are designed to provide guidance and help, but trying to encourage self-work and research. The evaluation was consistent with the methodology used and it has taken into account the scope of the analysis, giving priority to the metholology used even more than the results themselves.
\end{abstract}

Keywords: Transversal skill; Problem Analysis and Resolution; Grade of Energy Engineering; Advanced Nuclear Power Plants. 
Desarrollo de la competencia transversal de "Análisis y Resolución de Problemas” en la asignatura Centrales Nucleares Avanzadas

\begin{abstract}
Resumen
La asignatura "Centrales Nucleares Avanzadas" se imparte en $4^{\circ}$ curso del Grado de Ingeniero de la Energía de la Universitat Politècnica de València. Se trata del primer año de docencia y actualmente cuenta con un número reducido de alumnos matriculados, con formación previa en Energía Nuclear gracias a la asignatura troncal "Tecnología Nuclear". En la asignatura se aborda el diseño y el análisis de seguridad de reactores nucleares de fisión que se prevé que entren en funcionamiento comercial en un futuro próximo. Durante este curso se ha comenzado a trabajar en la competencia transversal de Análisis y Resolución de Problemas en las sesiones de prácticas y en algunas sesiones de teoría. Conscientes de la importancia del desarrollo de esta competencia, se han diseñado diferentes sesiones para resolver un problema (no ejercicio) complejo, en el que los alumnos deben involucrarse en la toma de decisiones. En este marco, se ha propuesto como problema el cálculo de una serie de parámetros de diseño de un reactor nuclear avanzado con un código de Monte Carlo. Se ha utilizado una sesión para resolver ejercicios como entrenamiento para enfrentarse al problema. Se ha propuesto un problema abierto y no completamente definido. Se han diseñado las sesiones proporcionando guías y ayuda, pero intentando fomentar el trabajo autónomo y la investigación. La evaluación ha sido coherente con la metodología empleada y se ha tenido muy en cuenta el alcance del análisis realizado, priorizándolo incluso más que los propios resultados.
\end{abstract}

Palabras clave: Competencia transversal; Análisis y resolución de problemas; Ingeniero de la Energía; Centrales Nucleares Avanzadas.

\title{
Introducción
}

La asignatura "Centrales Nucleares Avanzadas" se imparte en $4^{\circ}$ curso (B) del Grado de Ingeniero de la Energía de la Universitat Politècnica de València. Los estudiantes matriculados tienen formación previa en Energía Nuclear gracias a la asignatura troncal “Tecnología Nuclear”. En el presente curso, la asignatura presenta 6 alumnos matriculados. Este número reducido de alumnos permite trabajar en metodologías de aprendizaje que pueden ser muy eficientes pero que implican un gran esfuerzo docente. Debido al propio carácter de la asignatura, el uso de metodologías para trabajar la competencia transversal de “Análisis y Resolución de Problemas” puede ser muy efectivo. Dicha competencia se define como identificar, analizar y definir los elementos significativos que constituyen un

(c)) EY-NC-ND 2015, Universitat Politècnica de València

Congreso IN-RED (2015) 
problema para resolverlo con criterio y de forma efectiva [1]. Esta competencia está relacionada con conceptos como el pensamiento analítico y sistémico, la actitud proactiva, la orientación al logro, racionalidad y visión y perspectiva de futuro, entre otros [2, 3 y 4]. Se establecen tres niveles de dominio: l) Identificar y analizar un problema para generar alternativas de solución, aplicando los métodos aprendidos; 2) Utilizar su experiencia y criterio para analizar las causas de un problema y construir una solución más eficientes y eficaz; 3) Proponer y construir en equipo soluciones a problemas en diversos ámbitos, con una visión global. [1].

Los indicadores del primer nivel de dominio son los siguientes:

1. Identifica lo que es y no es un problema y toma la decisión de abordarlo.

2. Lee y/o escucha activamente. Hace preguntas para definir el problema planteado.

3. Recoge la información significativa que necesita para resolver los problemas en base a datos y no sólo a opiniones subjetivas y sigue un método lógico de análisis de la información.

4. Sigue un método lógico para identificar las causas de un problema y no quedarse en los síntomas.

5. Presenta diferentes opciones alternativas de solución ante un mismo problema y evalúa sus posibles riesgos y ventajas.

6. Diseña un plan de acción para la aplicación de la solución escogida.

Se ha decidido trabajar los indicadores 1, 2, 3, 4 y 6 del primer nivel de dominio de la comptencia. Para desarrollar esta competencia se ha propuesto a los alumnos la resolución de un problema complejo, la cual requerirá de 7 sesiones de trabajo en aula y trabajo autónomo por parte de los alumnos.

La evaluación de la asignatura incluye 4 métodos: una prueba escrita de respuesta abierta (40\% de peso), una prueba objetiva (20\%), un trabajo académico (20\%) y un trabajo de prácticas (20\%). La competencia de “Análisis y Resolución de Problemas" se evalúa mediante el trabajo de prácticas y de manera parcial, mediante la exposición correspondiente al trabajo académico.

\section{Objetivos}

El objetivo fundamental de este trabajo es desarrollar la competencia transvesal "Análisis y resolución de problemas" en la asignatura de "Centrales Nucleares Avanzadas".

Este objetivo general, se concreta en tres objetivos específicos:

1- Diseñar, planificar y ejecutar sesiones de teoría y prácticas encaminadas al trabajo de la competencia objeto de estudio. 
Desarrollo de la competencia transversal de “Análisis y Resolución de Problemas” en la asignatura Centrales Nucleares Avanzadas

2- Plantear un(os) problema(s) que representen retos realistas para los alumnos. Estos problemas deben pertenecer al campo de proximidad de sus intereses como estudiantes.

3- Establecer un sistema de evaluación acorde con la metodología, coherente y sólido.

\section{Desarrollo de la innovación}

En el presente curso y con el objetivo de trabajar la competencia transversal "Análisis y Resolución de Problemas", se ha analizado un problema en el ámbito de la Ingeniería Nuclear.

Se han planteado 4 sesiones prácticas (de 2,5 horas cada una) y 3 sesiones de teoría de aula (de 1,5 horas) con la siguiente estructuración:

Sesión 1: Descripción general del problema a resolver. En esta sesión se presenta el problema a los alumnos, haciendo hincapié en que hay información que no se dispone y ello obligará a tomar decisiones, evaluar las posibles simplificaciones, valorar el impacto de las simplificaciones en la validez del resultado, etc.

Sesión 2: Se plantea como una sesión de tránsito, es decir, una sesión dedicada exclusivamente a la resolución de ejercicios, a modo de entrenamiento. En la Figura 1 se muestran las geometrías correspondientes a algunos de los ejercicios propuestos como entrenamiento antes de abordar el problema propuesto en la asignatura. El objetivo principal de esta sesión es que los alumnos comiencen a conocer la herramienta y la potencialidad de la simulación. De manera transversal, en esta sesión se trabaja la competencia transversal "Instrumental específica" y se desarrolla de manera implícita la visión espacial para reproducir de manera adecuada geometrías 3D en representaciones bidimensionales. La planificación de la sesión es la siguiente: se presenta el programa de simulación y se describe brevemente la potencialidad de la herramienta. A continuación, se realiza un ejemplo guiado a modo de tutorial con el objetivo de mostrar el procedimiento de trabajo. En la segunda parte de la sesión, se proponen 4 ejercicios para que los alumnos conozcan los comandos del programa y adquieran soltura para afrontar posteriormente trabajos más complejos. En principio esta sesión no presenta grandes dificultades ni para los alumnos ni para los docentes porque es un reflejo de la metodología tradicional de trabajo a la que todos los implicados están familiarizados. 

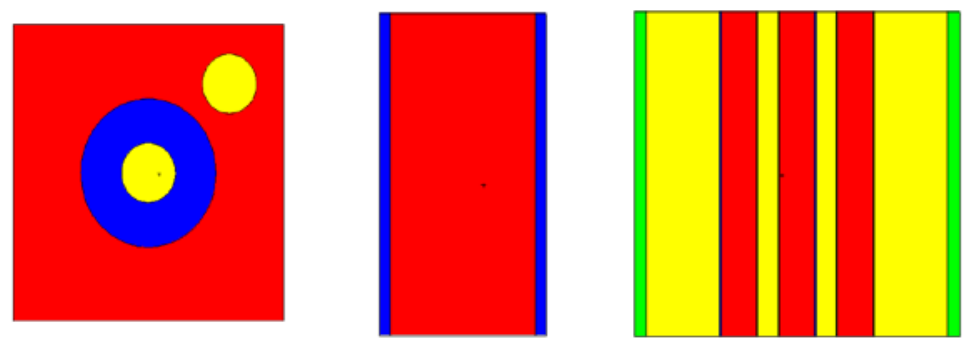

Fig. 1 Geometrías correspondientes a diferentes ejercicios de entrenamiento

Sesiones 3, 4 y 5: Búsqueda de información, modelización del núcleo de un reactor “Advanced Candu Reactor” (ACR) y toma de decisiones para simplificar el problema. Para cada sesión se plantea una serie de metas, que van aumentando en complejidad hasta alcanzar el objetivo último. En este caso en concreto, se han establecido las siguientes metas, como hitos a alcanzar al finalizar cada una de estas tres sesiones:

Meta 1. Modelizar un elemento combustible ACR. Para alcanzar esta meta, los alumnos deben realizar un trabajo previo a la sesión práctica. Este trabajo consiste en estudiar el manual del usuario del código informático que se utiliza, aconsejándose para ello seguir un tutorial. Además, se les proporciona unas directrices sobre lo que se va a diseñar en la práctica. Es este punto de especial relevancia para el óptimo desarrollo de la experiencia porque es entonces cuando los alumnos aprecian la complejidad de la tarea, la información de la que disponen y descubren que están frente a un problema, no un ejercicio. Esta meta (meta 1) está asignada a la sesión 3 y es determinante para la motivación de los alumnos, ya que descubren que son capaces de analizar los datos que les proporcionamos, capaces de determinar qué información necesitan y cuál es la información de la que no disponen. Es en este momento cuando comienza la discusión más enriquecedora del proceso: los estudiantes se hacen preguntas a sí mismos. ¿Es completamente necesaria la información que no dispongo? ¿Cómo afectará al resultado el asumir simplificaciones? ¿Será la simplificación asumible o invalidará el resultado? ¿Cómo me preparo para utilizar un programa a partir del manual y de unos ejercicios previos de preparación?

Se considera que en esta sesión es necesario que 2 profesores tutoricen a los 6 alumnos porque es en este momento en que los alumnos comienzan a dar sus primeros pasos para alcanzar la autonomía en el diseño. Se pide como evidencia del trabajo realizado, la entrega de un pequeño informe (2 ó 3 páginas) de los apartados del manual consultados, incluyendo una breve descripción del procedimiento general para definir la geometría del problema. También se solicita que incluyan una relación de las páginas web consultadas para la búsqueda de información. La parte relativa a la búsqueda de información tiene una importancia bastante relevante porque los alumnos llegan a la asignatura con hábitos de 
Desarrollo de la competencia transversal de “Análisis y Resolución de Problemas” en la asignatura Centrales Nucleares Avanzadas

búsqueda poco operativos, sin poder discernir entre información de calidad e información irrelevante o de poca fiabilidad. La sesión se completa proporcionando las guías necesarias para la elaboración de la geometría de un elemento combustible, tal y como se muestra en Figura 2.

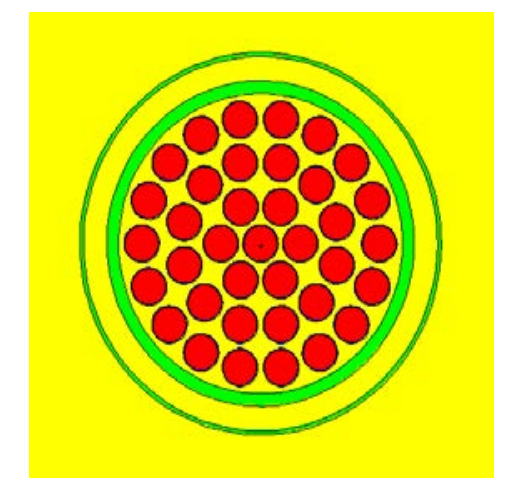

Fig. 2 Sección transversal de un elmento combustible

Meta 2 (sesión 4). Diseño del núcleo del reactor. Una vez realizado el modelo del elemento combustible, los alumnos deben ser capaces de modelar el núcleo utilizando una serie de herramientas para realizar repeticiones y mallados tridimensionales. De nuevo, el planteamiento es evitar proporcionar una "receta" para que la sigan sin hacerse preguntas. En este sentido, ésta es la sesión más complicada del curso porque les obliga a trabajar la visión espacial, trabajar con instrucciones muy técnicas de manuales especializados (en inglés) y de nuevo a realizarse la pregunta: ¿Cómo lo hago? ¿Es la mejor forma? ¿Estoy haciéndolo bien? Resulta curioso constatar que a medida que aumenta el grado de dificultad de la tarea, se incrementa el grado de motivación. Los autores de este trabajo hemos comprobado que algunos de los estudiantes, se toman la tarea como un reto. Sin embargo, también consideramos que hay que definir bien el alcance y los límites del problema, porque si se complica en exceso y no se consigue resolver, se puede producir el efecto contrario al buscado, es decir, la desmotivación. La Figura 3 muestra la geometría a la que los alumnos deben llegar al finalizar la sesión 4. 

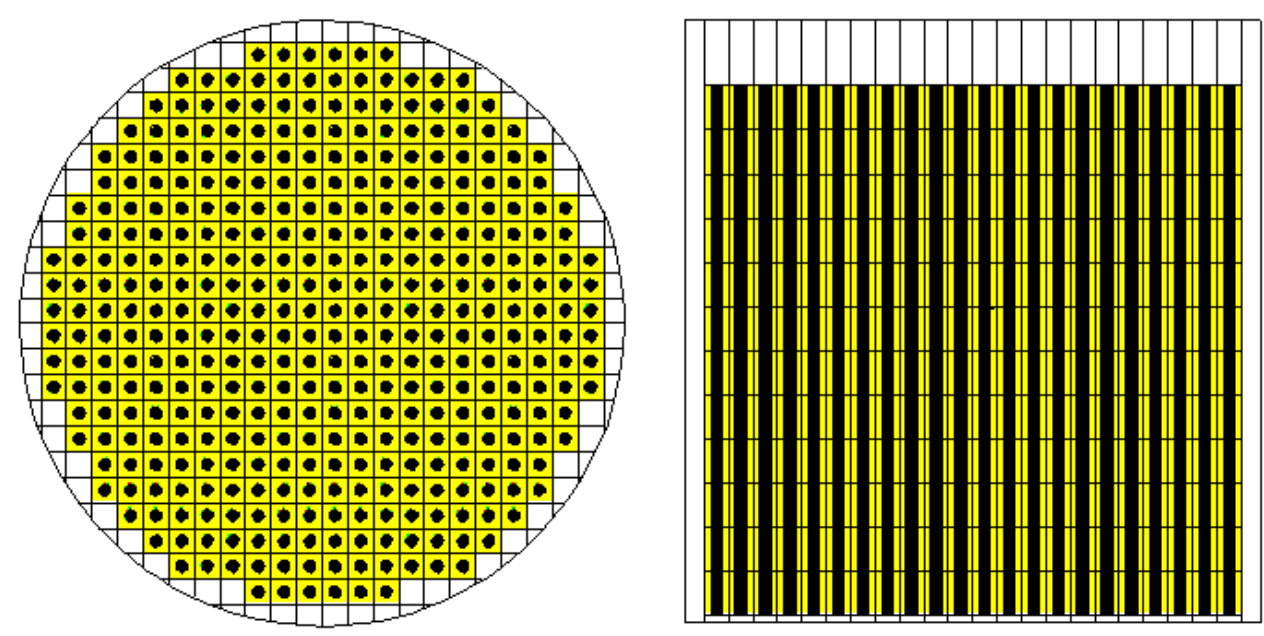

Fig. 3 Sección transversal y longitudinal del núcleo

Además de la geometría, la definición de los materiales que la conforman, representa otro reto para los alumnos. Deben relacionar conocimientos adquiridos previamente en otras asignaturas (materiales, metalúrgia, química, etc) con los requerimientos de los materiales en reactores nucleares. Como evidencia del trabajo realizado en esta sesión, los alumnos deben presentar un informe de 2 ó 3 páginas con el procedimiento seguido, la información consultada y el grado de consecución del objetivo de la sesión. La entrega de este tipo de evidencias resulta un poco sorprendente para los alumnos, ya que no están muy familiarizados con la realización de informes de seguimiento del trabajo. La ventaja adicional de la elaboración de estos mini-informes es que el alumno no pierde en ningún momento el hilo de la discusión ni la coherencia del trabajo.

Meta 3 (sesión 5). Análisis de resultados.

Esta es una de las sesiones más agradecidas y motivadoras de todas las planteadas. Por fin, después de 5 sesiones de 2,5 horas cada una, los alumnos obtienen resultados tras introducir en el código, el modelo desarrollado anteriormente. En esta sesión se les proporciona las herramientas necesarias para hacer cálculos de criticidad del reactor y se les da unas directrices generales sobre el tipo de información que podrían obtener si siguen trabajando por su cuenta en este problema. En este punto se presta especial atención al análisis crítico de los resultados, favorenciendo el debate y la discusión entre ellos. Recordemos que cada uno de los alumnos ha podido decidir qué información es más relevante y cuál es prescindible. La pública comparación de los resultados no pretende establecer un ránking

\section{(cc)) EY-NC-ND 2015, Universitat Politècnica de València}

Congreso In-Red (2015) 
Desarrollo de la competencia transversal de “Análisis y Resolución de Problemas” en la asignatura Centrales Nucleares Avanzadas

de las mejores simulaciones, sino fomentar la discusión y evitar los efectos negativos de hacer cálculos sin un análisis sistemático y científico del problema.

Sesión 6: Esta sesión se dedica a la exposición del trabajo a los compañeros de clase. Cada estudiante realiza una presentación en powerpoint (con una duración máxima de 20 min) con 10 minutos de preguntas por parte del profesor y de los compañeros.

De nuevo, se trata de una sesión motivadora, porque se muestra el fruto de un trabajo que ha requerido búsqueda de información, aprendizaje de un programa complejo, manejo de instrucciones técnicas en inglés, adopción de simplificaciones y por último obtención e interpretación de resultados. En esta sesión se trabaja la competencia transversal de la Comunicación Efectiva. Se intenta crear un clima serio pero lo suficientemente distendido para propiciar el debate y la discusión entre ellos y con el profesor. Se ha comprobado en este curso, que los alumnos se preguntan entre ellos demostrando una aceptable madurez de la información que han trabajado durante las últimas sesiones. A modo de prueba, este año los alumnos han asignado de manera anónima una puntuación a las exposiciones de los demás compañeros. Se ha constatado que los alumnos son, en muchas ocasiones, más críticos y extrictos con sus compañeros que lo es el propio profesor. De hecho, la calificación media asignada por los alumnos a los trabajos de sus compañeros es un punto inferior que la asignada por el profesor.

Después de analizar la experiencia, los profesores de la asignatura están estudiando la posibilidad de realizar durante el próximo curso una sesión de defensa de trabajos mediante la exposición de un póster. Esta posibilidad abre la puerta a nuevos retos que deben afrontar los alumnos: sintetización de la información, estructuración del discurso, trabajo de herramientas específicas de comunicación, etc.

Sesión 7: La evaluación busca guardar la coherencia con la metodología utilizada. De manera implícita, se ha realizado una evaluación cuasi-continua de la actividad durante las 6 sesiones anteriores. En esta última sesión, se realiza una prueba escrita en la que se pide al estudiante que justifique de manera argumentada una serie de afirmaciones relativas a la modelización del núcleo. Se está trabajando en una rúbrica para mejorar la evaluación de esta competencia en la asignatura.

Elaborar una prueba escrita de estas características es complicado por dos motivos: en primer lugar, las preguntas no deben quedarse en la mera teoría, es decir, deben orientarse en términos de relaciones, efectos, consecuencias, etc. En segundo lugar, esta evaluación debe ser coherente con la metodología de trabajo. Ello implica abandonar en buena medida la zona de confort de explicar únicamente la teoría y adentrarse en el desarrollo de

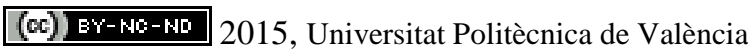

Congreso IN-RED (2015) 
competencias como el aplicación de pensamiento práctico y estudio de problemas contemporáneos. Esta asignatura permite relacionar muchos contenidos teóricos, con la actualidad, con el pensamiento crítico, con la sociedad, etc. Este trabajo, duro para ambas partes, profesores y alumnos, permite posteriormente ampliar la evaluación más allás de los límites de la teoría y los ejercicios.

De cualquier modo, los indicadores del primer nivel de dominio correspondiente a la competencia “Análisis y Resolución de Problemas" [1] deben ser convenientemente evaluados. Por este motivo, el 20\% de la calificación final de la asignatura se centra en la evaluación de esta competencia.

La correcta evaluación de estos indicadores implica realizar implícitamente una evaluación en cada una de las sesiones (7 en total) planteadas en la asignatura. Como ayuda a la sistematización de este método de evaluación, se ha comenzado a trabajar en una rúbrica de estos indicadores para intentar aumentar la objetividad en el cálculo del grado de consecución de la competencia. Después de cada una de las 7 sesiones, el profesor cumplimenta un formulario en el que aparecen los 5 indicadores objeto de estudio y una escala de baremación de 1 a 5 . A partir de la puntuación obtenida para los 5 indicadores se calcula el valor medio aritmético. Se repite la operación para el resto de las sesiones de prácticas y las de teoría (relacionadas con el trabajo del problema en estudio). Finalmente se obtiene una calificación a partir de la media aritmética de las 7 calificaciones. Esta calificación es la correspondiente al $20 \%$ de la calificación de la asignatura (correspondiéndose con la nota de prácticas). La exposición del trabajo tiene un peso total del $20 \%$ en la asignatura. De este $20 \%$, la mitad de la calificación se corresponde con la manera en la que el alumno relaciona el proceso de abordar y resolver el problema con los contenidos teóricos del propio problema. Es decir, el alumno debe exponer cómo ha resuelto el problema, qué simplificaciones ha realizado, cómo ha elaborado el plan para la resolución, qué fuentes de información ha utilizado, etc.

\section{Resultados}

Es difícil analizar los resultados de esta experiencia por varios motivos: En primer lugar, es el primer año de docencia de la asignatura y en segundo lugar, el número de alumnos es muy reducido como para tratar los resultados estadísticamente de manera rigurosa. Se cuenta, sin embargo con una encuesta de satisfacción de creación propia que se ha pasado a los alumnos en la que se pregunta su grado de acuerdo o desacuerdo ante determinadas afirmaciones:

-El entrenamiento con ejercicios ha sido adecuado para abordar con éxito un problema.

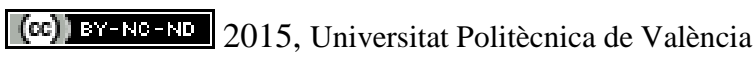

Congreso In-Red (2015) 
Desarrollo de la competencia transversal de “Análisis y Resolución de Problemas” en la asignatura Centrales Nucleares Avanzadas

-He sido capaz de estructurar un problema.

-He sido capaz de distinguir la información esencial de la irrelevante o prescindible. Me he enfrentado a situaciones cada vez más nuevas y abiertas.

-He sido capaz de resolver un problema.

-Me siento satisfecho con el trabajo realizado y con los conocimientos adquiridos. Soy consciente que he trabajado contenidos tecnológicos y he trabajado en una competencia aplicable a mi futura vida laboral.

Los resultados tras procesar los cuestionarios de los alumnos, es que todos han percibido en mayor o menor grado el significado de trabajar en un problema, pero absolutamente todos han valorado muy positivamente la metodología respecto de la tradicional. Por otro lado, otro resultado del trabajo es la introducción de una evalación acorde con la metodología. Se ha reformulado la prueba escrita de manera que se prima el razonamiento y la argumentación frente a la memorización de datos. Se considera que este cambio en la evaluación es un resultado muy interesante de cara a la futura profundización del trabajo en ésta u otras competencias.

\section{Conclusiones}

La metodología utilizada para trabajar la competencia transversal de “Análisis y resolución de problemas” en la asignatura “Centrales Nucleares Avanzadas” parece ser adecuada después de analizar los resultados de la encuesta de satisfacción de los alumnos de elaboración propia. La metodología seguida, planteando un problema complejo, abierto, con falta de información y con otra información redundante o irrelevante fuerza al estudiante a abandonar la comodidad de los caminos establecidos y limitados, y poner en marcha recursos que normalmente no utilizan. Esta vertiente, amplia y libre, se ha intentado compatibilizar con una vertiente más cerrada y rígida: el uso de herramientas de modelización. El resultado es satisfactorio tanto para los estudiantes como para el docente.

\section{Referencias}

[1] VILLA, A. y POBLETE, M. Aprendizaje basado en compentencias. Una propuesta para la evaluación de las competencias genéricas.

[2] ESCRIBANO, A., DEL VALLE, A., El aprendizaje basado en problemas. Una propuesta metodológica en Educación Superior., Narcea, S.A. de Ediciones, 2008-

[3] SANZ DE ACEO, M. L., Competencias cognitivas en Educación Superior, Narcea, Madrid, 2010.

[4] CHANG, R. y KELLY, P. Resolución de problemas. Barcelona: Granica, 1996.

(c)) EY-NC-ND 2015, Universitat Politècnica de València 
S. Gallardo y S. Carlos

[5] WECKWERTH, E., Problem solving and Decision Making. Minneapolis: University of Minnesota 1990.

(cc) EY-NC-ND 2015, Universitat Politècnica de València

Congreso In-Red (2015) 